\title{
Mutations in the facilitative glucose transporter GLUT10 alter angiogenesis and cause arterial tortuosity syndrome
}

\author{
Paul J Coucke ${ }^{1}$, Andy Willaert ${ }^{1}$, Marja W Wessels ${ }^{2}$, Bert Callewaert ${ }^{1}$, Nicoletta Zoppi ${ }^{3}$, Julie De Backer ${ }^{1}$, \\ Joyce E Fox ${ }^{4}$, Grazia M S Mancini ${ }^{2}$, Marios Kambouris ${ }^{5}$, Rita Gardella ${ }^{3}$, Fabio Facchetti ${ }^{6}$, Patrick J Willems ${ }^{7}$, \\ Ramses Forsyth ${ }^{8}$, Harry C Dietz ${ }^{9}$, Sergio Barlati ${ }^{3}$, Marina Colombi ${ }^{3}$, Bart Loeys ${ }^{1}$ \& Anne De Paepe ${ }^{1}$
}

Arterial tortuosity syndrome (ATS) is an autosomal recessive disorder characterized by tortuosity, elongation, stenosis and aneurysm formation in the major arteries owing to disruption of elastic fibers in the medial layer of the arterial wall ${ }^{1}$.

Previously, we used homozygosity mapping to map a candidate locus in a 4.1-Mb region on chromosome 20q13.1 (ref. 2).

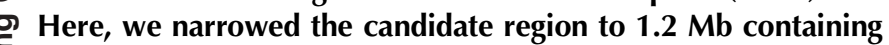
seven genes. Mutations in one of these genes, SLC2A10, encoding the facilitative glucose transporter GLUT10, were identified in six ATS families. GLUT10 deficiency is associated with upregulation of the TGF $\beta$ pathway in the arterial wall, a finding also observed in Loeys-Dietz syndrome, in which aortic aneurysms associate with arterial tortuosity ${ }^{3}$. The identification of a glucose transporter gene responsible for altered arterial morphogenesis is notable in light of the previously suggested link between GLUT10 and type 2 diabetes ${ }^{4,5}$. Our data could provide new insight on the mechanisms causing microangiopathic changes associated with diabetes and suggest that therapeutic compounds intervening with TGF $\beta$ signaling represent a new treatment strategy.

Facilitative glucose transporters (GLUTs), encoded by a family of $S C L 2 A$ genes, are responsible for the uptake of several monosaccharides, including glucose, fructose, mannose, galactose and glucosamine. So far, mutations in two of these genes have been linked to genetic disorders with intuitive relevance to altered glucose metabolism. Heterozygous mutations in SLC2A1 cause a defect of glucose transport into the brain, resulting in an epileptic encephalopathy with low spinal-fluid glucose levels ${ }^{6}$. Homozygous mutations in SLC2A2 have been shown to cause Fanconi-Bickel syndrome, characterized by hepatorenal glycogen accumulation, nephropathy and diarrhea ${ }^{7}$, whereas heterozygous mutations in this gene result in non-insulin dependent diabetes mellitus ${ }^{8,9}$.
We report that loss-of-function mutations in a third member of the SLC2A family, SLC2A10, cause arterial tortuosity syndrome (ATS; OMIM 208050), an autosomal recessive condition ${ }^{1}$ characterized by tortuosity of the large and medium-sized arteries (Fig. 1a), often resulting in death at young age. Other typical features include aneurysms of large arteries and stenosis of the pulmonary artery, in association with facial features (Fig. 1b) and several connective tissue manifestations. Histopathological findings include fragmentation of the elastic fibers in the tunica media of the large arteries (Fig. 1c) ${ }^{10-13}$. Previously, homozygosity mapping in 21 members of two consanguineous families with ATS originating from Morocco (family 1) and Italy (family 4; Fig. 1d) assigned the gene to chromosome 20q13.1 (ref. 2). Subsequently, this localization was confirmed in four smaller families originating from Morocco (families 2 and 3) and the Middle East (family 5 and 6). Key recombinants delineated a candidate linkage interval of $4.1 \mathrm{Mb}$ between markers D20S836 and D20S109. We performed further fine mapping in three families (families 1-3) originating from the same region in Morocco, under the assumption that one recessive ancestral mutation might have caused ATS in these families. Families 1 and 2, but not family 3, shared haplotypes between markers D20S888 and $\mu$ SAT11 (Fig. 1e), a region of $1.2 \mathrm{Mb}$ containing seven genes (SLC13A3, TP53RK, SLC2A10, EYA2, PRKCBP1, NCOA3, SULF2) and one pseudogene (RPL35AP). We sequenced these genes directly and identified homozygous mutations (deletion, nonsense, missense) in the SLC2A10 gene in all six families (Fig. 2a,b). In families 1 and 2, we found a homozygous nonsense mutation $510 \mathrm{G} \rightarrow \mathrm{A}(\mathrm{W} 170 \mathrm{X})$ in all clinically affected individuals. All affected individuals in families 3 and 4 were homozygous for frameshift mutations 961delG (V321fsX391) and 1334delG (G445fsX484), respectively. Both mutations result in a premature stop codon. The affected individuals from families 5 and 6 shared the same homozygous missense mutation, $243 \mathrm{C} \rightarrow \mathrm{G}(\mathrm{S} 81 \mathrm{R})$. Both families had a common haplotype between markers $\mu$ SAT1 and $\mu$ SAT7, indicating a

\footnotetext{
${ }^{1}$ Center for Medical Genetics, Ghent University, B-9000 Ghent, Belgium. ${ }^{2}$ Department of Clinical Genetics, Erasmus University Medical Center, 3015 GD Rotterdam, The Netherlands. ${ }^{3}$ Division of Biology and Genetics, Department of Biomedical Sciences and Biotechnology, University of Brescia, Brescia 25123 , Italy. ${ }^{4}$ Department of Pediatrics, North Shore University Hospital, Manhasset, New York 11030, USA. ${ }^{5}$ Yale University School of Medicine, New Haven, Connecticut 06510, USA. ${ }^{6}$ Department of Pathology, University of Brescia, Brescia, Italy. ${ }^{7}$ GENDIA, B-2000 Antwerp, Belgium. ${ }^{8}$ Department of Pathology, Ghent University, B-9000 Ghent, Belgium. ${ }^{9}$ McKusick-Nathans Institute of Genetic Medicine and Howard Hughes Medical Institute, Johns Hopkins School of Medicine, Baltimore 21205, Maryland, USA. Correspondence should be addressed to P.J.C. (paul.coucke@ugent.be).
} 
a
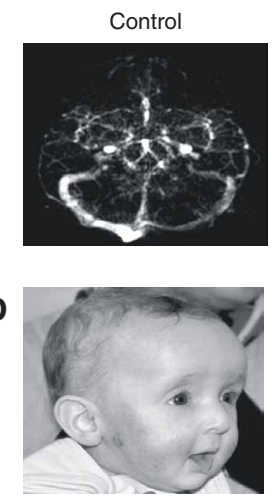

C

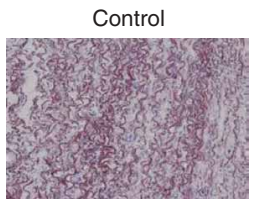

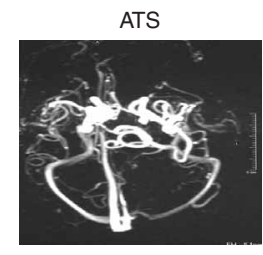

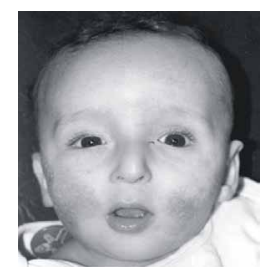

ATS

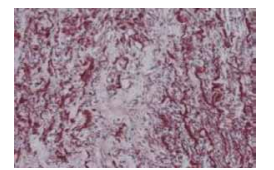

d
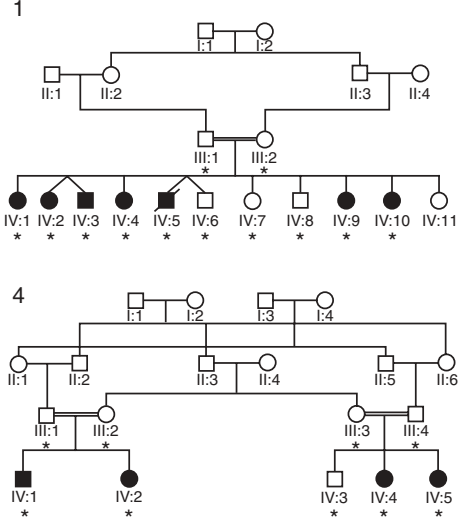

e

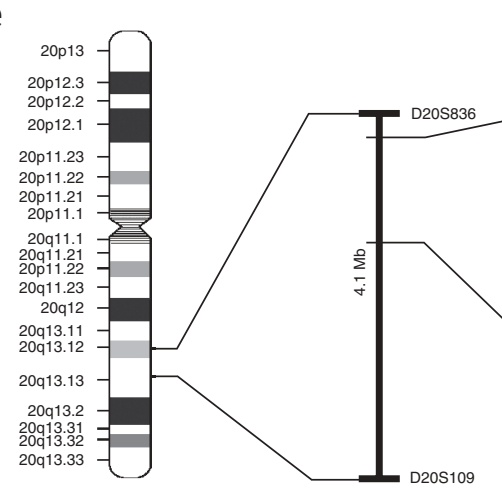

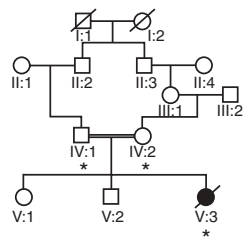

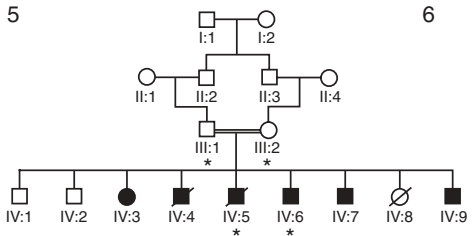

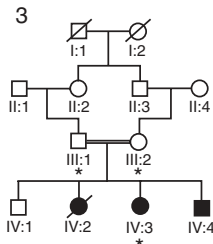

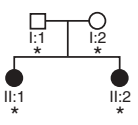

Figure 1 Clinical anomalies in ATS and pedigrees. (a) MR angiography showing typical arterial tortuosity of the cerebral arteries in an individual with ATS (IV:4 in family 1) in comparison with a healthy aged-matched control. (b) Typical facial phenotype with micrognathia, elongated face, down-slanting palpebral fissures, blepharophimosis and a beaked nose (individual IV:4 in family 1). (c) Organization of elastic fibers in a control and in an individual with ATS, as shown by orcein staining. Aorta elastic laminae in the media of an individual with ATS are coarser, less abundant and more disorganized than in control aorta. Magnification: 400×. (d) Pedigree structure of the six ATS families. Symbols: circle, female; square, male; open symbol, unaffected; filled symbol, affected; slash line, deceased; double relationship line, consanguinity. Asterisks indicate that DNA, fibroblasts or both are available. (e) Ideogram of chromosome 20 showing the initial linkage interval, the final candidate region and haplotypes for chromosome $20 q 13.1$ markers in the candidate region in families 1-3.

founder mutation in these families (data not shown). We assume that the latter mutation causes disease on the basis of the following arguments: (i) Ser81 is evolutionarily strictly conserved in GLUT10 (Fig. 2b); (ii) an uncharged amino acid is changed to a positively charged amino acid in the third transmembrane domain and (iii) the mutation was absent in 200 control chromosomes. All parents of affected individuals (in families 1-6) were heterozygous for the respective mutations.

The presence of homozygous loss-of-function mutations in at least four ATS families identifies SLC2A10 as the gene responsible for ATS. The gene contains five exons and encodes GLUT10, a 541-residue glucose transporter ${ }^{14-16}$. Human GLUT10 has been shown to facilitate D-glucose, D-galactose and 2-deoxy-D-glucose transport when expressed in Xenopus laevis oocytes ${ }^{4}$. GLUT10 is an outlier within the GLUT family because of its longer exofacial loop and differences in motif characteristics for glucose transporters, suggesting that GLUT10 may have additional functions, different from other GLUT family members ${ }^{4,5}$.

Tissue expression analysis has uncovered a widespread distribution of SLC2A10 mRNA, mainly in liver, pancreas and adipose tissue $e^{4,5,17}$. We studied mRNA and protein expression of GLUT10 in cultured skin fibroblasts and vascular smooth muscle cells (VSMCs) from individuals affected with ATS and from controls. Quantitative PCR (Q-PCR) of samples derived from individuals with ATS homozygous for premature stop codon mutations demonstrated a near-absence of SLC2A10 mRNA in VSMCs as well as in fibroblasts (Fig. 2c), as expected by virtue of clearance of mutant transcripts by the nonsensemediated mRNA decay (NMD) pathway. We observed normal SLC2A10 mRNA expression in samples derived from an individual with ATS homozygous for the $243 \mathrm{C} \rightarrow \mathrm{G}$ missense mutation. We observed (peri)nuclear localization of GLUT10 in normal individuals, but there was no detectable GLUT10 signal in individuals with ATS, as shown by immunofluorescence analysis of cultured skin fibroblasts and VSMCs (Fig. 2d). An additional argument to suggest a nuclear localization of GLUT10 is the low dissociation constant $\left(K_{\mathrm{m}}=0.3 \mathrm{mM}\right)$, which is compatible with the glucose concentration in the cytoplasm ${ }^{4}$.

The SLC2A10 gene has previously been considered as a candidate gene for diabetes because of its function in glucose transport and its map position, which coincides with a type 2 diabetes locus ${ }^{4,5}$. However, a causal role for SLC2A10 in diabetes has not been demonstrated $^{18,19}$. Theoretically, homozygous mutations in SLC2A10 could lead to ATS, whereas heterozygous mutations could lead to diabetes, analogous to the situation where homozygous mutations in SLC2A2 
a

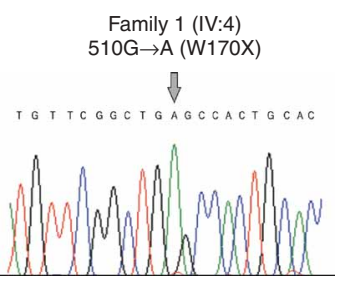

Family 3 (IV:3) 961delG (V321fs)

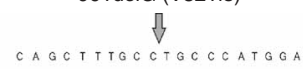

CAGCIITGCGTGCCCATGGA

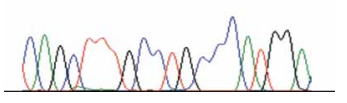

Family 5 (IV:5) $243 C \rightarrow G(S 81 R)$

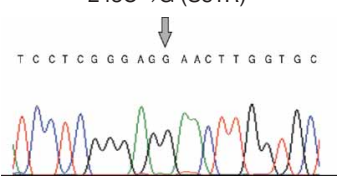

C

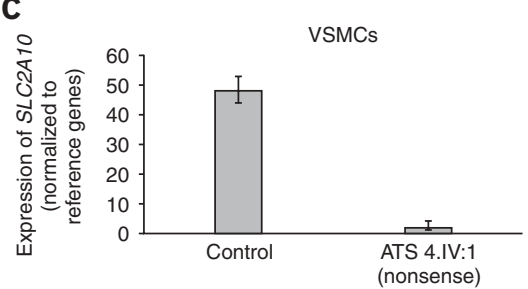

Family 2 (V:3)

$510 \mathrm{G} \rightarrow \mathrm{A}(\mathrm{W} 170 \mathrm{X})$ $\sqrt{ }$

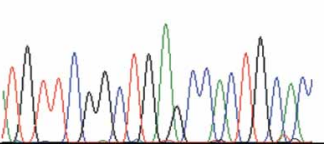

Family 4 (IV:5) 1334delG (G445fs) $\sqrt{ }$
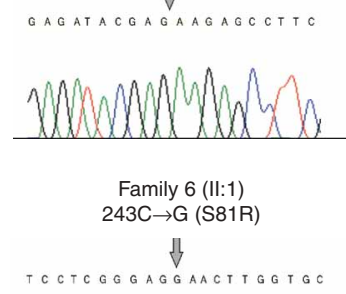
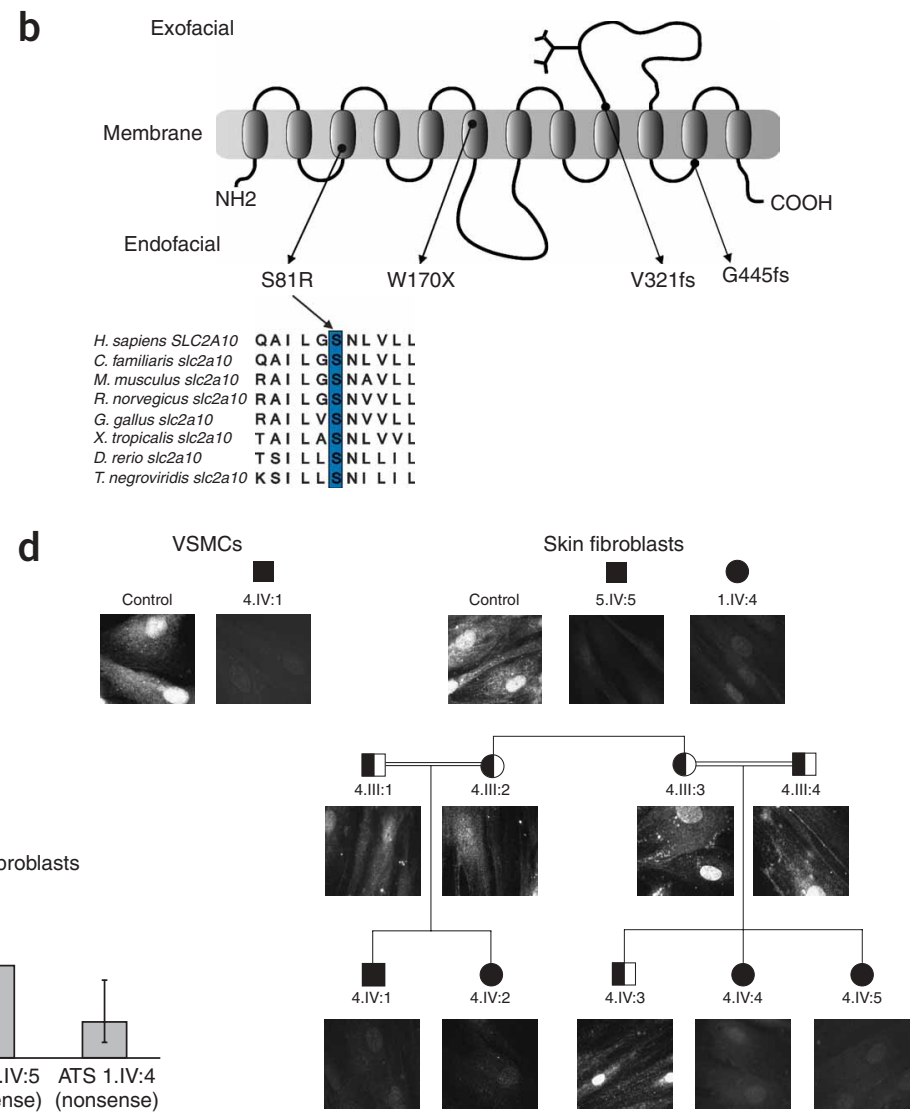

Figure 2 SLC2A1O (GLUT10) mutation and expression data. (a) SLC2A10 mutations identified in families 1-6. (b) Location of GLUT10 mutations at a schematic representation of the GLUT10 protein. GLUT10 contains 12 hydrophobic transmembrane domains (ovals) with a hydrophilic endofacial loop between transmembrane domains 6 and 7 and a large exofacial loop containing a potential N-linked glycosylation site between transmembrane domains 9 and 10. Evolutionary conservation of the substituted amino acid observed in families 5 and 6 in GLUT10 is shown. (c,d) SLC2A10 (GLUT10) expression in the control and in individuals with ATS. (c) mRNA expression of SLC2A10 as determined by Q-PCR in VSMCs and skin fibroblasts. In VSMCs in individuals with ATS, the level of mRNA was severely reduced. In skin fibroblasts, the individual carrying a homozygous nonsense mutation also showed a significant reduction $(P<0.05)$ compared with the control, but the individual homozygous for a missense mutation did not show any reduction. Bars indicate the 95\% confidence interval of the mean expression level. (d) Immunofluorescence analysis of GLUT10 in VSMCs and skin fibroblasts. Expression of GLUT10 was nearly absent in VSMCs and fibroblasts from individuals with ATS, as compared with the control. The fluorescence signal in heterozygous individuals was approximately half that of the controls. Magnification: 1,000x.

lead to Fanconi-Bickel syndrome ${ }^{7}$ and heterozygous mutations to diabetes $^{8}$. However, this hypothesis is unlikely, given that we did not observe an increased frequency of diabetes in the heterozygotes from the ATS families.

There is substantial phenotypic overlap between ATS and a newly identified genetic condition called Loeys-Dietz syndrome (LDS; OMIM 609192) that associates arterial tortuosity with aneurysm formation ${ }^{3}$. Other findings in common between the two conditions include arachnodactyly, joint laxity or contractions, microretrognathia, hypertelorism, cleft palate and/or bifid uvula (Table 1). LDS is caused by heterozygous loss-of-function mutations in the genes encoding the type 1 or type 2 TGF $\beta$ receptors (TGFBR1 or TGFBR2). This leads to a paradoxical increase in TGF $\beta$ signaling in the arterial wall, as evidenced by increased phosphorylation and nuclear translocation of Smad2 (pSmad2), a downstream effector of the TGF $\beta$ signaling pathway, and increased expression of downstream targets of TGF $\beta$ such as connective tissue growth factor (CTGF) and collagens ${ }^{3}$. Because of the clinical overlap with LDS, we investigated whether the TGF $\beta$ pathway is involved in the pathogenesis of ATS.
Immunostaining for pSmad2 and CTGF (Fig. 3a) in the arterial wall of an individual with ATS demonstrated increased signal intensity compared with control specimens, similar to the increase observed in individuals with $\mathrm{LDS}^{3}$. In agreement with the in vivo observations, QPCR measurements showed a significantly higher steady-state mRNA expression level for CTGF (Fig. 3b) in cultured VSMCs of the individual with ATS compared with controls $(P<0.05)$, indicative of upregulation of TGF $\beta$ signaling.

The mechanisms by which mutations in SLC2A10 lead to TGF $\beta$ activation are unclear. Notably, the expression of decorin, a proteoglycan inhibitor of TGF $\beta$ signaling ${ }^{20}$, is regulated by a defined glucose response element in its gene promoter ${ }^{21}$. Therefore, we studied the expression of decorin in cultured VSMCs of individuals with LDS, individuals with ATS and controls (Fig. 4). Decorin expression was severely reduced in cultured VSMCs of individuals with ATS as compared with controls, as shown by immunofluorescence staining (Fig. 4a). Q-PCR experiments using VSMCs confirmed the reduced expression of decorin mRNA in individuals with ATS (Fig. 4b). The specific decrease of decorin expression in individuals with ATS, in contrast to individuals with LDS, might indicate divergent mechanisms 
Table 1 Clinical comparison of individuals with ATS and individuals with LDS

\begin{tabular}{|c|c|c|}
\hline Symptoms & ATS & LDS \\
\hline \multicolumn{3}{|l|}{ Arterial anomalies } \\
\hline Tortuosity & +++ & +++ \\
\hline Aneurysms & + & +++ \\
\hline Stenosis a. pulmonalis & ++ & 0 \\
\hline Aneurysm a. pulmonalis & + & ++ \\
\hline Skin laxity & +++ & + \\
\hline \multicolumn{3}{|l|}{ Skeletal anomalies } \\
\hline Contractures & ++ & ++ \\
\hline Pectus deformity & + & ++ \\
\hline Joint laxity & +++ & +++ \\
\hline Arachnodactyly & + & ++ \\
\hline \multicolumn{3}{|l|}{ Facial anomalies } \\
\hline Hypertelorism & + & +++ \\
\hline Cleft palate, bifid uvula & ++ & +++ \\
\hline Microretrognathia & +++ & ++ \\
\hline
\end{tabular}

for upregulation of TGF $\beta$ signaling in these two conditions. Notably, we did not observe any differences in expression between fibroblasts of individuals with ATS and those of healthy controls (data not shown). Given the (peri)nuclear localization of GLUT10, a decrease in intracellular glucose and failure of glucose-mediated transcriptional upregulation of the decorin promoter seems to be unique to ATS. In contrast, primary alterations in the TGF $\beta$ receptors lead to increased TGF $\beta$ signaling in LDS. In order to determine the specificity of the decorin response, we monitored the expression of versican, a

\section{a}
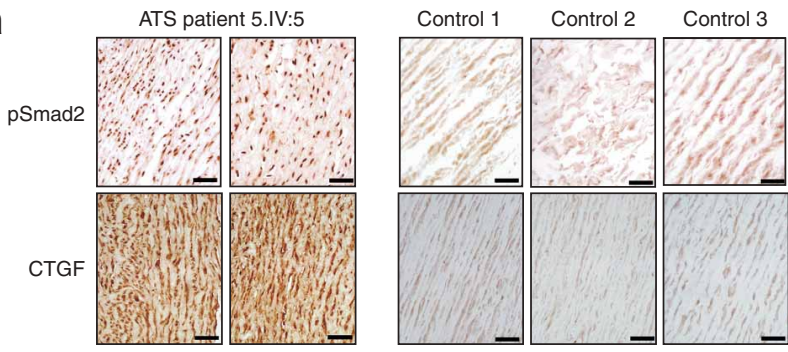

b

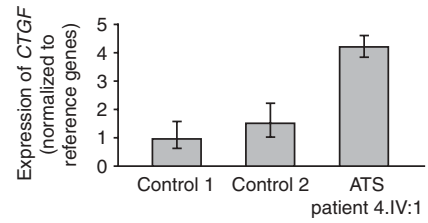

Figure 3 Immunostaining and Q-PCR analysis for phosphorylated Smad2 and CTGF in arterial tissue. (a) Three controls and individual 5.IV:5 with ATS were examined. Note the increased intensity of nuclear phosphorylated Smad2 and extracellular CTGF. Scale bars, $10 \mu \mathrm{m}$. (b) Expression levels of CTGF in VSMCs of affected individual IV: 1 of family 4 and two controls in steady-state conditions, as measured by Q-PCR. CTGF shows fourfold higher expression in the individual with ATS. Bars indicate the 95\% confidence interval of the mean expression level.
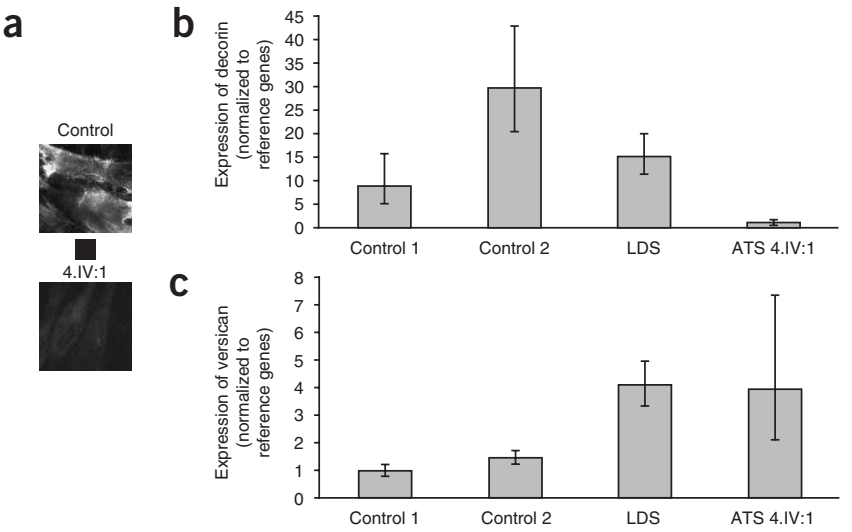

Figure 4 Immunofluorescence and Q-PCR analysis of decorin and versican in VSMC. (a) In individual 4.IV:1 with ATS, expression of decorin is nearly absent, as compared with control VSMCs. Magnification: 1,000 $\times$. (b,c) Q-PCR analysis for (b) decorin and (c) versican in VSMCs of an individual with ATS compared with an individual with LDS and two controls. The expression of decorin mRNA in the individual with ATS is significantly lower $(P<0.05)$ than in the LDS patient and the controls, whereas the expression of versican is significantly higher $(P<0.05)$ in the individual with ATS and the individual with LDS compared with controls. Bars indicate the $95 \%$ confidence interval of the mean expression level.

proteoglycan that is known not to be strongly regulated by glucose but whose expression is driven by TGF $\beta^{22}$. As predicted from our pathogenetic model, samples from patients with either ATS or LDS showed increased expression of versican (Fig. 4c). An inhibitory role of versican on elastic fiber assembly has been proposed ${ }^{23}$, perhaps providing a mechanism for failed elastogenesis in both ATS and LDS.

Although TGF $\beta$ signaling is disturbed in cells and connective tissue derived from individuals with ATS, other mechanisms leading to abnormal matrix deposition cannot be excluded. Impaired uptake or transport of other monosaccharides could hinder glycosylation events important for the production of mature glycoproteins and proteoglycans, essential structural components of the arterial wall and connective tissue in general.

We were surprised to identify a glucose transporter gene responsible for a connective tissue disorder. No other connective tissue disorder, with the exception of pseudoxanthoma elasticum (caused by a mutation in $A B C C 6$ ), is known to be caused by a transporter protein defect $^{24}$. No clear function of the ABCC6 transporter has been identified, and the underlying pathogenic mechanism leading to disturbed elastin homeostasis in PXE is unknown. Insights derived from the study of ATS may prove relevant to other disorders related to failed intracellular transport of glucose. Indeed, the microangiopathic changes and fibrosis seen in diabetic retinopathy, nephropathy and peripheral vascular disease correlate with increased TGF $\beta$ signaling ${ }^{25}$. Diabetesassociated arteriolar tortuosity is seen in tissues undergoing postnatal angiogenesis, including the retinal and coronary microcirculations, perhaps recapitulating events occurring on a broader scale during embryogenesis in ATS. These data suggest that antagonism of TGF $\beta$ signaling may contribute to therapeutic advances in a wide variety of genetically determined and acquired disorders, including ATS.

\section{METHODS}

Patients. Appropriate informed consent, including specific consent to publish the photos in Figure 1b, was obtained from all patients involved in the study. Detailed clinical descriptions of five ATS families, except for family 6, have been 
published previously ${ }^{1,12,26}$. DNA was extracted from peripheral blood and/or cultured skin fibroblasts. Skin fibroblasts were cultured in Dulbecco's modified Eagle's medium (DMEM) supplemented with $15 \%$ fetal bovine serum (FBS) in the presence of antibiotics. Vascular smooth muscle cells (VSMCs) were obtained from the aortic media from individual IV:1 with ATS in family 4 and from an individual with LDS (TGFBR1 mutation, R487P) ${ }^{3}$ and were cultured in smooth muscle basal medium supplemented 5\% FBS, $0.2 \%$ fibroblast growth factor (FGF), $0.1 \%$ insulin, $0.1 \%$ epidermal growth factor (EGF), and $0.1 \%$ gentamicin sulfate/amphotericin. Paraffin-embedded aortic tissue was available only from individual IV:5 in family 5 .

Microsatellite and sequence analysis. Microsatellite markers in the ATS linkage region on chromosome 20q13.1 (ref. 2) were taken from the Marshfield map or designed based on the simple tandem repeat finder in the University of California Santa Cruz genome browser ( $\mu$ SAT1-11). We carried out genotyping on an Applied Biosystems Prism 3100 Genetic Analyzer (Applied Biosystems). The data were processed using Genescan software (Applied Biosystems).

We amplified all coding exons from all seven genes in the ATS linkage region (SLC13A3, TP53RK, SLC2A10, EYA2, PRKCBP1, NCOA3, SULF2) by PCR using intronic primers and additional exonic primers for larger exons (Supplementary Table 1 online). Sequencing was performed using the BigDye v3.1 ET terminator cycle sequencing kit (Applied Biosystems). Sequencing reactions were loaded onto an Applied Biosystems Prism 3100 Genetic Analyzer.

Immunostaining. For GLUT10 and decorin analysis in VSMCs and skin fibroblasts by immunofluorescence microscopy, we grew $1 \times 10^{5}$ VSMCs or skin fibroblasts from controls and from individuals with ATS for $72 \mathrm{~h}$ in complete medium. To analyze GLUT10, the cells were washed in PBS, permeabilized in $0.5 \%$ Triton X-100 and 3\% paraformaldehyde for $2 \mathrm{~min}$, fixed for $20 \mathrm{~min}$ in $3 \%$ paraformaldehyde, incubated for $30 \mathrm{~min}$ at room temperature $\left(21^{\circ} \mathrm{C}\right)$ with $5 \%$ BSA/PBS and incubated overnight at $4{ }^{\circ} \mathrm{C}$ with $20 \mu \mathrm{g} \mathrm{ml}^{-1}$ polyclonal antibody to GLUT10 (Alpha Diagnostic). To analyze decorin levels, the cells were fixed in 3\% paraformaldehyde for $10 \mathrm{~min}$, washed twice for $5 \mathrm{~min}$ in PBS and incubated for $40 \mathrm{~min}$ at room temperature $\left(21{ }^{\circ} \mathrm{C}\right)$ with $20 \mu \mathrm{g} \mathrm{ml}^{-1}$ monoclonal antibody to decorin (clone 115402, R\&D Systems). Next, the VSMCs and fibroblasts were incubated for $1 \mathrm{~h}$ at room temperature $\left(21{ }^{\circ} \mathrm{C}\right)$ with rhodamine-conjugated anti-rabbit IgG $(1: 50$ in $1 \%$ BSA/PBS) and anti-mouse IgG (1:100 in $1 \%$ BSA/PBS), respectively; washed in PBS; mounted in 1:1 PBS-glycerol solution on glass slides and photographed with a Zeiss Axiovert 10S/H fluorescence microscope. Quanti50 tative evaluation of the fluorescence was performed as previously reported ${ }^{27}$. For GLUT10, quantitative evaluation was repeated on 20 randomly selected cells for each cell strain. Images were digitized to measure the cell and the nuclear areas and the integrated optical density (IOD) corresponding to the fluorescence signals.

For immunohistochemical staining for CTGF and pSmad2 in arterial tissue, we selected representative specimens of formalin-fixed, paraffin-embedded arterial media of three healthy control individuals and one individual with ATS. From these specimens, 5 - $\mu \mathrm{m}$ thick paraffin sections were cut, deparaffinized and rehydrated. These tissues were pretreated with a protease-1 enzymatic solution (Ventana). For immunohistochemical analysis, we used antibodies directed against pSmad2 and CTGF (Alpha Diagnostic, Cell Signaling Technology and Abcam, respectively) and previously described methods ${ }^{3}$. Light microscopy was performed on an Olympus BX45 microscope.

Q-PCR. RNA was isolated using the RNeasy Mini Kit (Qiagen), and cDNA was synthesized using SuperScript II Reverse Transcriptase Kit with random hexamer primers (Invitrogen) in a total volume of $20 \mu \mathrm{l}$. Two microliters of cDNA (1:10 dilution) and $250 \mathrm{nM}$ gene-specific primers were used with the Q-PCR Core Kit for SYBR Green I (Eurogentec) for Q-PCR on a GeneAmp 5700 Sequence Detector (Applied Biosystems). The Q-PCR program consisted of 40 cycles with $15 \mathrm{~s}$ at $95^{\circ} \mathrm{C}$ and $1 \mathrm{~min}$ at $60{ }^{\circ} \mathrm{C}$, followed by a dissociation run to determine melting curves. We carried out all reactions in duplicate and normalized them to the geometric mean of three reference genes (GAPDH, HPRT1 and YWHAZ). We used fibroblasts from controls and individuals with ATS and VSMCs, obtained from aortic media from two controls, from individual IV:1 with ATS (from family 4) and from an individual with LDS $^{3}$. We grew cells in $6-\mathrm{cm}$ dishes to $80 \%$ confluence. Expression levels were determined in three independent experiments for each cell line. Differential gene expression was considered significant when the difference was at least $50 \%$ and the $95 \%$ confidence interval of the mean expression levels did not overlap (equivalent to $P<0.05$ ).

Accession codes. GenBank: SLC2A10 cDNA, NM_030777; SLC2A10 coding region, NT_011362.

\section{Note: Supplementary information is available on the Nature Genetics website.}

\section{ACKNOWLEDGMENTS}

We are indebted to the families and patients for their interest and cooperation. We thank C.E. Catsman-Berrevoets for referring family 1. We thank P. Van Acker and L.A. Myers for technical assistance. This study was supported by the Fund for Scientific Research, Flanders (Belgium); Ghent University; the Ministero dell'Istruzione, dell'Università e della Ricerca, Fondo per gli Investimenti della Ricerca di Base 2001; Fondazione Cariplo; Fondazione Berlucchi; the William Smilow Center for Marfan Syndrome Research; the Howard Hughes Medical Institute and the US National Institutes of Health. B.C. and B.L. are research assistant and senior clinical investigator, respectively, and are supported by the Fund for Scientific Research, Flanders. J.D.B. is a research fellow from Ghent University.

\section{COMPETING INTERESTS STATEMENT}

The authors declare that they have no competing financial interests.

\section{Published online at http://www.nature.com/naturegenetics}

Reprints and permissions information is available online at http://npg.nature.com/ reprintsandpermissions/

1. Wessels, M.W. et al. Three new families with arterial tortuosity syndrome. Am. J. Med. Genet. A 131, 134-143 (2004)

2. Coucke, P.J. et al. Homozygosity mapping of a gene for arterial tortuosity syndrome to chromosome 20q13. J. Med. Genet. 40, 747-751 (2003).

3. Loeys, B.L. et al. A syndrome of altered cardiovascular, craniofacial, neurocognitive and skeletal development caused by mutations in TGFBR1 or TGFBR2. Nat. Genet. 37, 275-281 (2005).

4. Dawson, P.A. et al. Sequence and functional analysis of GLUT10: a glucose transporter in the Type 2 diabetes-linked region of chromosome 20q12-13.1. Mol. Genet. Metab. 74, 186-199 (2001)

5. McVie-Wylie, A.J., Lamson, D.R. \& Chen, Y.T. Molecular cloning of a novel member of the GLUT family of transporters, SLC2a10 (GLUT10), localized on chromosome 20q13.1: a candidate gene for NIDDM susceptibility. Genomics 72, 113-117 (2001).

6. Seidner, G. et al. GLUT-1 deficiency syndrome caused by haploinsufficiency of the blood-brain barrier hexose carrier. Nat. Genet. 18, 188-191 (1998).

7. Santer, R. et al. Mutations in GLUT2, the gene for the liver-type glucose transporter, in patients with Fanconi-Bickel syndrome. Nat. Genet. 17, 324-326 (1997).

8. Tanizawa, Y. et al. Variability of the pancreatic islet beta cell//iver (GLUT 2) glucose transporter gene in NIDDM patients. Diabetologia 37, 420-427 (1994).

9. Sakamoto, O. et al. Mutation analysis of the GLUT2 gene in patients with FanconiBickel syndrome. Pediatr. Res. 48, 586-589 (2000).

10. Ertugrul, A. Diffuse tortuosity and lengthening of the arteries. Circulation 36 400-407 (1967).

11. Beuren, A.J., Hort, W., Kalbfleisch, H., Muller, H. \& Stoermer, J. Dysplasia of the systemic and pulmonary arterial system with tortuosity and lengthening of the arteries. A new entity, diagnosed during life, and leading to coronary death in early childhood. Circulation 39, 109-115 (1969).

12. Pletcher, B.A. et al. Four sibs with arterial tortuosity: description and review of the literature. Am. J. Med. Genet. 66, 121-128 (1996).

13. Franceschini, P., Guala, A., Licata, D., Di Cara, G. \& Franceschini, D. Arterial tortuosity syndrome. Am. J. Med. Genet. 91, 141-143 (2000).

14. Barrett, M.P., Walmsley, A.R. \& Gould, G.W. Structure and function of facilitative sugar transporters. Curr. Opin. Cell Biol. 11, 496-502 (1999).

15. Joost, H.G. \& Thorens, B. The extended GLUT-family of sugar/polyol transport facilitators: nomenclature, sequence characteristics, and potential function of its novel members (review). Mol. Membr. Biol. 18, 247-256 (2001).

16. Scheepers, A., Joost, H.G. \& Schurmann, A. The glucose transporter families SGLT and GLUT: molecular basis of normal and aberrant function. JPEN J. Parenter. Enteral Nutr. 28, 364-371 (2004).

17. Wood, I.S., Hunter, L. \& Trayhurn, P. Expression of Class III facilitative glucose transporter genes (GLUT-10 and GLUT-12) in mouse and human adipose tissues. Biochem. Biophys. Res. Commun. 308, 43-49 (2003).

18. Andersen, G. et al. Genetic variation of the GLUT10 glucose transporter (SLC2A10) and relationships to type 2 diabetes and intermediary traits. Diabetes 52, 2445-2448 (2003). 
19. Mohlke, K.L. et al. Evaluation of SLC2A10 (GLUT10) as a candidate gene for type 2 diabetes and related traits in Finns. Mol. Genet. Metab. 85, 323-327 (2005).

20. Fischer, J.W., Kinsella, M.G., Levkau, B., Clowes, A.W. \& Wight, T.N. Retroviral overexpression of decorin differentially affects the response of arterial smooth muscle cells to growth factors. Arterioscler. Thromb. Vasc. Biol. 21, 777-784 (2001).

21. Wahab, N.A., Parker, S., Sraer, J.D. \& Mason, R.M. The decorin high glucose response element and mechanism of its activation in human mesangial cells. J. Am. Soc Nephrol. 11, 1607-1619 (2000).

22. Schonherr, E., Jarvelainen, H.T., Sandell, L.J. \& Wight, T.N. Effects of platelet-derived growth factor and transforming growth factor-beta 1 on the synthesis of a large versican-like chondroitin sulfate proteoglycan by arterial smooth muscle cells. J. Biol. Chem. 266, 17640-17647 (1991).

23. Wight, T.N. \& Merrilees, M.J. Proteoglycans in atherosclerosis and restenosis: key roles for versican. Circ. Res. 94, 1158-1167 (2004).

24. Le Saux, O. et al. Mutations in a gene encoding an ABC transporter cause pseudoxanthoma elasticum. Nat. Genet. 25, 223-227 (2000).

25. Chiarelli, F., Santilli, F. \& Mohn, A. Role of growth factors in the development of diabetic complications. Horm. Res. 53, 53-67 (2000).

26. Gardella, R. et al. Exclusion of candidate genes in a family with arterial tortuosity syndrome. Am. J. Med. Genet. A 126, 221-228 (2004).

27. Colombi, M. et al. Matrix assembly induction and cell migration and invasion inhibition by a 13-amino acid fibronectin peptide. J. Biol. Chem. 278, 14346-14355 (2003). 